\title{
A J oint Analysis of the KOSPI 200 Option and ODAX Option Markets Dynamics
}

\author{
Ji Cao* \\ Wolfgang Härdle* \\ Julius Mungo*
}

\section{$\underline{v}$ \\ u \\ $\bar{x}$}

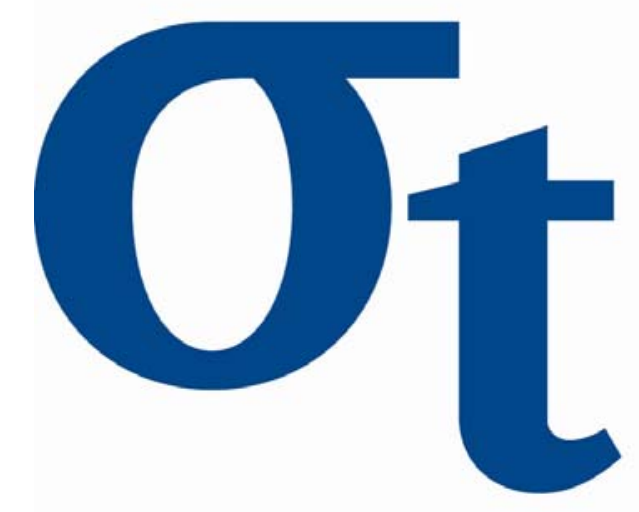

9)

$\forall$

6

*Humboldt-Universität zu Berlin, Germany

This research was supported by the Deutsche Forschungsgemeinschaft through the SFB 649 "Economic Risk". 


\title{
A Joint Analysis of the KOSPI 200 Option and ODAX Option Markets Dynamics*
}

\author{
Ji Cao † Wolfgang K. Härdle $\ddagger$ Julius Mungo ${ }^{\S}$
}

\begin{abstract}
As a function of strike and time to maturity the implied volatility estimation is a challenging task in financial econometrics. Dynamic Semiparametric Factor Models (DSFM) are a model class that allows for the estimation of the implied volatility surface (IVS) in a dynamic context, employing semiparametric factor functions and time-varying loadings. Because financial asset volatilities move over time, across assets and over markets, this paper analyses volatility interaction between German and Korean stock markets. As proxy for the volatility, factor loadings series derived from a DSFM application on option prices are employed. We examine volatility transmission between the markets under the vector autoregressive (VAR) model framework. Our results show that a shock in the volatility of one market may not translate directly into greater uncertainty in another market and it is unlikely that portfolio investors can benefit from diversification among these markets due to cointegration.
\end{abstract}

Keywords: implied volatility surface, dynamic semiparametric factor model, VAR, cointegration

JEL classification: C14; G12

${ }^{*}$ The financial support from the Deutsche Forschungsgemeinschaft via SFB 649 "Ökonomisches Risiko", Humboldt-Universität zu Berlin is gratefully acknowledged.

${ }^{\dagger}$ Corresponding author. Institute for Statistics and Econometrics of HumboldtUniversität zu Berlin, Spandauer Straße 1, 10178 Berlin, Germany. Email: ji.cao@wiwi.huberlin.de.

${ }^{\ddagger}$ Center for Applied Statistics and Economics, Humboldt-Universität zu Berlin, Spandauer Straße 1, 10178 Berlin, Germany.

${ }^{\S}$ Institute for Statistics and Econometrics of Humboldt-Universität zu Berlin, Spandauer Straße 1, 10178 Berlin, Germany. 


\section{Introduction}

As one of the most important parameters in financial markets, volatility has attracted a lot of attention. In derivatives pricing and hedging, volatility is a key issue and because volatility is unknown, one studies the implied volatility (IV) which is derived from the Black-Scholes formula (BS). By plugging the observed option price into the BS formula it is straightforward to calculate the implied volatility. Implied volatility is seen as a mapping from time $t$, moneyness $\kappa$ (a measure of strike) and time to maturity $\tau$, $\widehat{\sigma}:(t, \kappa, \tau) \rightarrow \widehat{\sigma}_{t}(\kappa, \tau)$.

Calculating implied volatilities at different strikes and maturities yields an implied volatility surface (IVS). One challenge in IVS modelling is that the data have a degenerated design: due to market conventions, maturities only have a small number of values such as one, two, three, six, nine and twelve months to expiry on the date of issue. This fact leads to a string structure of observations in IVS. As time passes these strings change their shape and location randomly. Figure 1 displays implied volatility of the ODAX on 2 May and 2 June, 2003. The implied volatilities on these two days show different patterns. On 2 May, the largest implied volatility is about 1, and it is about 2 on 2 June. There are three different expiration times on 2 May but eight on 2 June.

Dynamic semiparametric factor models are designed to capture these features of the IVS with low-dimensional factor functions and time-varying factor loadings. The IVS is approximated by unknown factor functions moving in a finite dimensional function space. The finite dimensional fits are obtained in the local neighbourhood of strikes and maturities, for which implied volatilities are recorded on the specific day. Surface estimation and dimension reduction is achieved in one single step. This approach can be seen as a combination of functional principal component analysis, nonparametric curve estimation and backfitting for additive models. The DSFM is generally expressed as

$$
Y_{t, j}=m_{0}\left(X_{t, j}\right)+\sum_{l=1}^{L} Z_{t, l} m_{l}\left(X_{t, j}\right)+\varepsilon_{t, j},
$$

where $Y_{t, j}$ is the log-implied volatility for option $j$ on day $t, t$ is an index of day $(t=1, \ldots, T)$ and $j$ is an index of options on day $t(j=1, \ldots, J) . X_{t, j}$ is two-dimensional covariables, $X_{t, j}=\left(\kappa_{t, j}, \tau_{t, j}\right)$ with $\kappa_{t, j}$ the moneyness (we define moneyness $\kappa_{t, j} \stackrel{\text { def }}{=} \frac{K_{t, j}}{F_{t, j}}$, where $K_{t, j}$ is the strike and $F_{t, j}$ is the future price.) and $\tau_{t, j}$ time to maturity. $m_{l}$ is time-invariant factor function and 

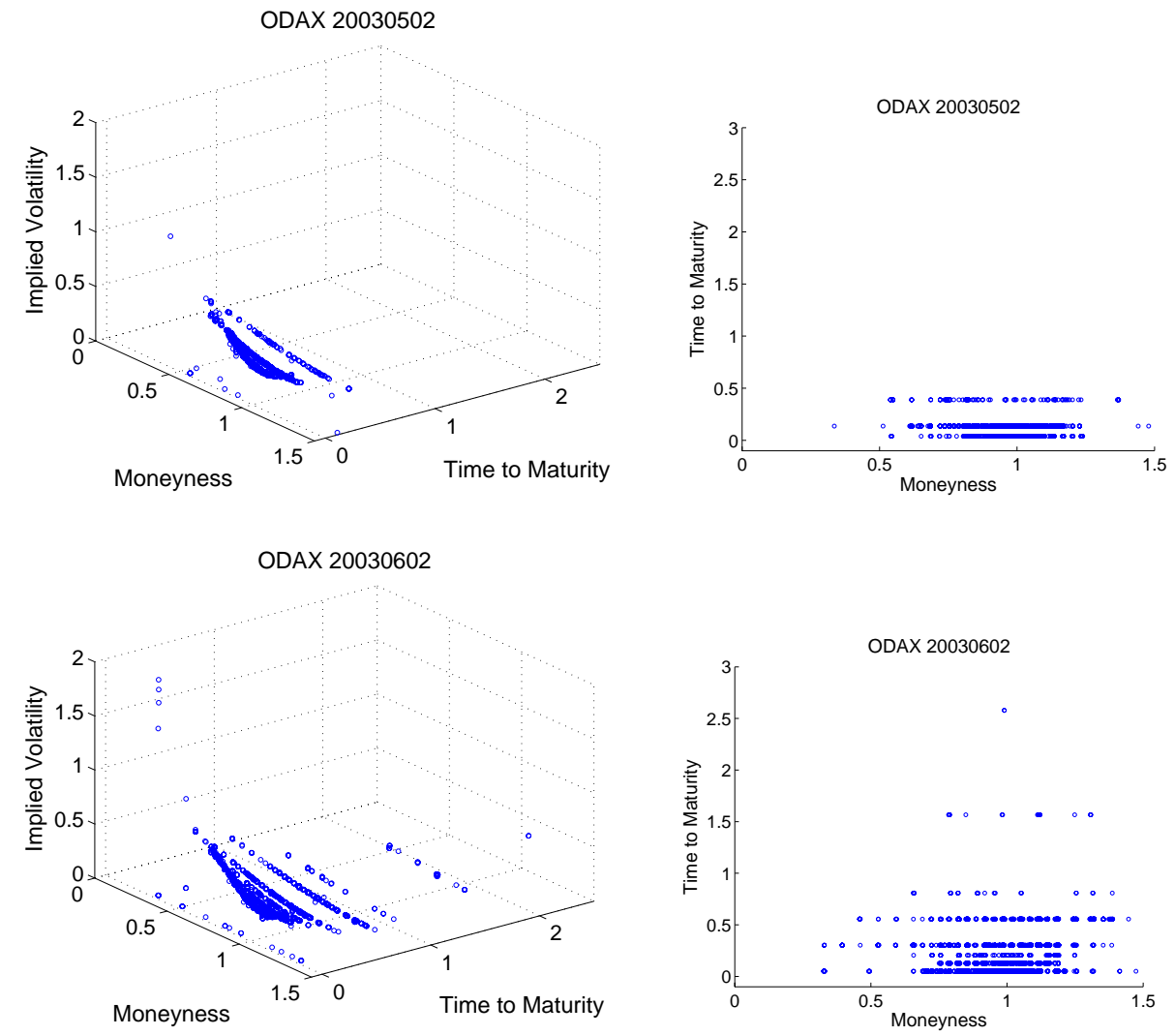

Figure 1: Upper left: implied volatility of ODAX on 2 May, 2003. (moneyness lower left axis, time to maturity lower right axis). Upper right: Data design of ODAX on 2 May, 2003. Lower left: implied volatility of ODAX on 2 June, 2003. Lower right: Data design of ODAX on 2 June, 2003. 
$Z_{t, l}$ is loading for factor $m_{l} . \varepsilon_{t, j}$ is an unknown error term with $E\left(\varepsilon_{t, j}\right)=0$ and $E\left(\varepsilon_{t, j}^{2}\right)<\infty$. After estimating unknown factor function $m_{l}$ and corresponding loading $Z_{t, l}$, implied volatility can be calculated given a desired point $\left(\kappa_{t, j}, \tau_{t, j}\right)$.

In a study related to the DSFM estimation, Park et al. (2009) have shown that the covariance structure of the estimated loading series converges in probability to the covariance structure of the unobservable loading series. This indicates that the additional error incurred by using $\widehat{Z}_{t}$ instead of $Z_{t}$ is negligible for large $T$. This asymptotic equivalence of the autocovariance structures carries over to classical estimation and testing procedures within the VAR model framework.

This paper applies the DSFM to estimate IVS of the Korean Stock Index (KOSPI 200) options and the ODAX. The estimation result is discussed and a joint analysis between two markets is studied. The paper is organised as follows: Section 2 describes data preparation and implied volatility calculation. The DSFM model fit is discussed in Section 3. Section 4 presents a joint analysis of the KOSPI option and the ODAX factor loading series dynamics. Conclusion is presented in section 5 .

\section{Data and Implied volatility calculation}

The data set contains tick statistics on KOSPI 200 option from the Korea Exchange and the ODAX from Eurex over the period from January 2003 to December 2003. The KOSPI 200 (Korea Composite Stock Price Index) is the index of 200 major companies' stocks traded on the Stock Market Division of the Korea Exchange. It is one of the most actively traded indices in the world. The Korea Exchange (KRX) is by transactional volume the largest derivatives exchange in the world. Deutscher Aktien IndeX 30 (German stock index) is a Blue Chip stock market index consisting of the 30 major German companies traded on the Frankfurt Stock Exchange. Its option ODAX is traded on Eurex (Germany and Switzerland) which is by far the world's largest international market organiser for the trading and settlement of futures and options on shares and share indices, as well as of interest rate derivatives. Both KOSPI 200 option data and ODAX data are recorded per transaction, that is, each observation contains information on option price, strike price, settlement time, maturity, etc.

One of the problems we face when calculating the implied volatility is the intraday underlying price. In Fengler et al. (2007) the intraday underlying price is used for IVS estimation. However, neither KOSPI data nor ODAX 
data in our case contains underlying price. In Fengler et al. (2007) the intraday future price within one-minute intervals is used to derive the underlying price. While for ODAX data corresponding future prices can be found in our case, we have not obtained any information of intraday futures price for KOSPI 200.

Another approach to obtain the intraday underlying price is to use put-call parity:

$$
C_{t}=P_{t}+S_{t}-D_{t}-K e^{-r \tau},
$$

where $C_{t}$ and $P_{t}$ are prices of European call and put options on the same underlying with the same strike price $K$ and same maturity $T$. $D_{t}$ is the discounted value of dividend paid by underlying during the time to maturity $\tau=T-t . r$ and $S_{t}$ are the interest rate and underlying price, respectively. Based on corresponding put and call option prices, the underlying price $S_{t}$ can be derived.

Unfortunately, there are only a few cases where pair of corresponding call and put contracts can be found in our data. Thus, intraday implied volatility cannot be calculated under these circumstances.

While it is difficult to find intraday underlying prices for both markets, acquiring closing prices is relatively easy, therefore, instead of using intraday prices, we use the closing prices in our analysis. Together with the last traded contract for each series of options - options with the same type, same maturity, same strike and traded on the same day, a closing implied volatility is calculated. Last traded contracts are extracted from the whole data set according to their settlement time. Many of these last traded contracts are settled between 14:00 and 15:15 hrs on the Korea Exchange and between 17:00 and 19:00 hrs on the Eurex - both near to the close time of their underlying markets. (On a normal trading day, trading of the KOSPI 200 option at the Korean Exchange ends at 15:15 hrs. On regular trading days, ODAX trading ends at 17:30 hrs with a post trading phase ending at 21:00 hrs.) At the same time, it is, however, also possible that the last traded contract is settled as early as 9:15 hrs - shortly after the market opens, thus an implied volatility based on this contract may not reflect the state of the market at close. Strictly speaking, using last traded contracts, together with closing underlying prices does not necessarily lead to closing implied volatility. To improve accuracy, some adjustments or smoothing techniques should be used in further study.

To calculate implied volatility, the interest rate for the KOSPI 200 option is the daily Korean treasury bill rate, for ODAX it is the daily EURIBOR rate in the same period. 
After extracting the last traded contracts from the whole data set, our sample size reduces and we are left with only about 40 observations per day. A linear interpolation is used in order to represent the data on a regular grid. Options on the same day and with the same maturity are grouped together and interpolated with respect to the moneyness grid $\kappa \in[0.8,1.2]$ with 1000 points. Furthermore, call option and put option are interpolated separately.

A new problem arises here: for both the KOSPI 200 option and the ODAX markets, implied volatilities calculated from put options and call options are different, even if they have the same strike, same maturity and same spot. In Fengler et al. (2007) a correction algorithm is used to obtain an adjusted underlying price, based on which, the put and call implied volatilities are the same. This algorithm (3) is based on the future price formula and put-call parity.

$$
\tilde{S}_{t}=e^{-r_{F}\left(T_{F}-t\right)} F_{t}+\Delta D_{t, T_{H}, T_{F}}
$$

where $\tilde{S}_{t}$ is the adjusted underlying price, $r_{F}$ is the interest rate used in deriving the underlying price from the future price, $T_{F}$ is the future's maturity time, $F_{t}$ is the future price, $T_{H}$ isthe option's maturity time, $\Delta D_{t, T_{H}, T_{F}}$ is the dividend difference calculated from put-call parity (2) and the future price formula $F_{t}=e^{r_{F}\left(T_{F}-t\right)} S_{t}-\Delta D_{t, T_{F}}, \Delta D_{t, T_{F}}$ is the dividend used for calculating future price.

However, it is a different situation in our case. Firstly, our underlying price is not obtained from the future price, but directly from the closing price in the underlying markets. Secondly, as stated before, there are only a few corresponding put and call options in our case, thus put-call parity cannot be used. Consequently, we don't apply algorithm (3) in our case. Instead, after interpolating put and call implied volatilities separately, we take the average of these two implied volatility series. Our algorithm of interpolation and average taking is described in detail as follows:

1. For each day, options (implied volatilities) with the same maturity are grouped together as $I V_{t, j}^{C}\left(\kappa_{m}\right)$ and $I V_{t, j}^{P}\left(\kappa_{n}\right) . t$ is day index. $j$ is group index on this day - index for different maturity. $C$ is call and $P$ is put. $\kappa_{m}$ and $\kappa_{n}$ are moneyness for calls and puts respectively, $m=1, \ldots, M, n=1, \ldots, N$.

2. For each group of these options $I V_{t, j}^{C}\left(\kappa_{m}\right)$ and $I V_{t, j}^{P}\left(\kappa_{n}\right)$,

(a) if both the number of calls $\sum_{m=1}^{M} m \geq 3$ and the number of puts $\sum_{n=1}^{N} n \geq 3$, call implied volatility and put implied volatility are 
linearly interpolated separately with respect to moneyness $\kappa$ on grid $\kappa \in[0.8,1.2]$ with 1000 points.

$$
I V_{t, j}^{C}\left(\kappa_{l}\right)=\frac{\kappa_{l}-\kappa_{m}}{\kappa_{m+1}-\kappa_{m}} I V_{t, j}^{C}\left(\kappa_{m+1}\right)+\frac{\kappa_{m+1}-\kappa_{l}}{\kappa_{m+1}-\kappa_{m}} I V_{t, j}^{C}\left(\kappa_{m}\right)
$$

and

$$
I V_{t, j}^{P}\left(\kappa_{l}\right)=\frac{\kappa_{l}-\kappa_{n}}{\kappa_{n+1}-\kappa_{n}} I V_{t, j}^{P}\left(\kappa_{n+1}\right)+\frac{\kappa_{n+1}-\kappa_{l}}{\kappa_{n+1}-\kappa_{n}} I V_{t, j}^{P}\left(\kappa_{m}\right),
$$

where $\kappa_{l}$ is desired moneyness point, $l \in[1,1000], \kappa_{l} \in[0.8,1.2]$. $\kappa_{m}$ and $\kappa_{m+1}$ are two closest observations to $\kappa_{l}$, with $\kappa_{m}<\kappa_{l}<$ $\kappa_{m+1}$. If $\max \left(\kappa_{m}\right)<\kappa_{l}$ or $\kappa_{l}<\min \left(\kappa_{m}\right)$, a constant extrapolation is used. For $\kappa_{n}$ and $\kappa_{n+1}$ is the same.

Then, an average of $I V_{t, j}^{C}\left(\kappa_{l}\right)$ and $I V_{t, j}^{P}\left(\kappa_{l}\right)$ is taken as the final implied volatility,

$$
I V_{t, j}\left(\kappa_{l}\right)=\frac{I V_{t, j}^{C}\left(\kappa_{l}\right)+I V_{t, j}^{P}\left(\kappa_{l}\right)}{2}
$$

(b) if only the number of calls $\sum_{m=1}^{M} m \geq 3$, while the number of puts $\sum_{n=1}^{N} n<3$, call implied volatilities are interpolated with (4). Put implied volatilities are dropped. Final implied volatilities are these interpolated call implied volatilities,

$$
I V_{t, j}\left(\kappa_{l}\right)=I V_{t, j}^{C}\left(\kappa_{l}\right)
$$

(c) similarly, if only the number of puts $\sum_{n=1}^{N} n \geq 3$, but the number of calls $\sum_{m=1}^{M} m<3$, put implied volatilities are interpolated with (5). Call implied volatilities are dropped. Final implied volatilities are these interpolated put implied volatilities,

$$
I V_{t, j}\left(\kappa_{l}\right)=I V_{t, j}^{P}\left(\kappa_{l}\right)
$$

(d) if both the number of calls $\sum_{m=1}^{M} m<3$ and the number of puts $\sum_{n=1}^{N} n<3$, final implied volatilities are the original observations,

$$
\begin{aligned}
& I V_{t, j}\left(\kappa_{m}\right)=I V_{t, j}^{C}\left(\kappa_{m}\right) \\
& I V_{t, j}\left(\kappa_{n}\right)=I V_{t, j}^{P}\left(\kappa_{n}\right) .
\end{aligned}
$$



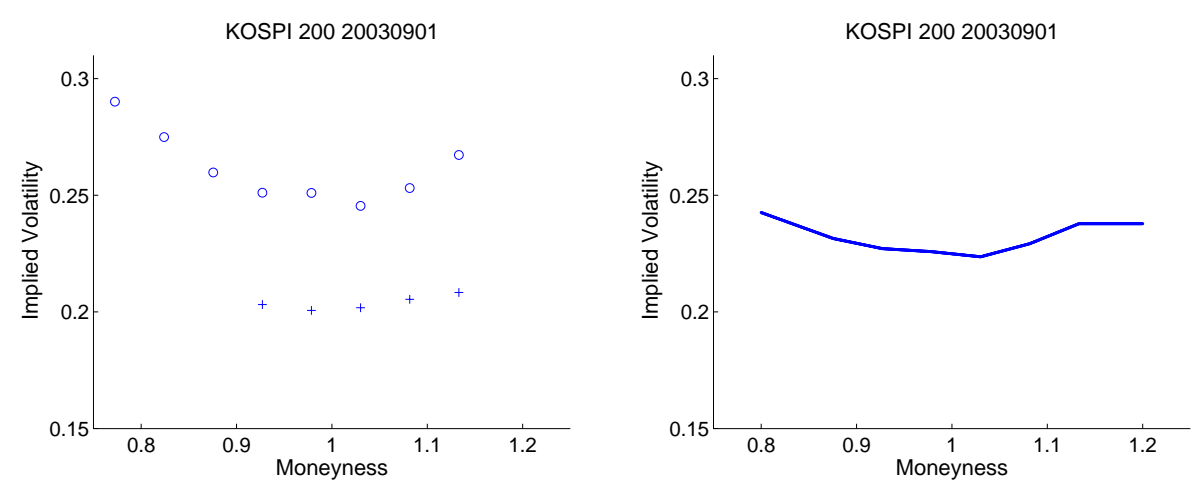

Figure 2: Implied volatility of last traded KOSPI 200 options with 102 days to maturity on 1 September, 2003. Left panel: original data, before interpolation. Call options are "+", put options are "o". Right panel: with linear interpolation and averaging.

Figure 2 is an example of one group of KOSPI 200 option data on 1 September, 2003. These contracts have 102 days to maturity. The left panel shows the original data - the last traded contracts taken from the entire data set. The right panel is based on the left panel, with interpolating call and put options separately and then averaging.

\section{Model fit}

The estimates $\widehat{m}_{l}(l=0, \ldots, L)$ and $\widehat{Z}_{t, l}(t=1, \ldots, T ; l=1, \ldots, L)$ are defined as minimisers of the following least squares criterion $\left(\widehat{Z}_{t, 0} \stackrel{\text { def }}{=} 1\right)$ :

$$
\sum_{t=1}^{T} \sum_{j=1}^{J_{i}} \int\left\{Y_{t, j}-\sum_{l=0}^{L} \widehat{Z}_{t, l} \widehat{m}_{l}(u)\right\}^{2} K_{h}\left(u-X_{t, j}\right) d u .
$$

Here, $K_{h}$ denotes a two-dimensional product kernel, $K_{h}(u)=k_{h_{1}}\left(u_{1}\right) \times$ $k_{h_{2}}\left(u_{2}\right), h=\left(h_{1}, h_{2}\right)$, based on the one-dimensional kernel $k_{h}(v) \stackrel{\text { def }}{=} h^{-1} k\left(h^{-1} v\right)$.

In (10) the minimisation runs over all the functions $\widehat{m}_{l}: \mathbb{R}^{2} \rightarrow \mathbb{R}$ and all values $\widehat{Z}_{t, l} \in \mathbb{R}$. In order to derive an estimator of the DSFM we proceed in the following way. (For the technical details we refer to Borak et al. (2005), Fengler et al. (2007) and Park et al. (2009).) For an $l^{\prime}$ with $1 \leq l^{\prime} \leq L$, for $\delta>0$ and a function $g$, we replace in (10) $\widehat{m}_{l^{\prime}}$ by $\widehat{m}_{l^{\prime}}+\delta g$ and take the first 
derivative with respect to $\delta$ at the point $\delta=0$. This yields

$$
\sum_{t=1}^{T} \sum_{j=1}^{J_{t}} \int\left\{Y_{t, j}-\sum_{l=0}^{L} \widehat{Z}_{t, l} \widehat{m}_{l}(u)\right\} \widehat{Z}_{t, l^{\prime}} K_{h}\left(u-X_{t, j}\right) d u=0 .
$$

Since the equation holds for all functions $g$, it implies that for $1 \leq l^{\prime} \leq L$ and for all $u$

$$
\sum_{t=1}^{T} \sum_{j=1}^{J_{t}}\left\{Y_{t, j}-\sum_{l=0}^{L} \widehat{Z}_{t, l} \widehat{m}_{l}(u)\right\} \widehat{Z}_{t, l^{\prime}} K_{h}\left(u-X_{t, j}\right)=0 .
$$

By replacing $\widehat{Z}_{t, l^{\prime}}$ by $\widehat{Z}_{t, l^{\prime}}+\delta$ in (10) and again taking derivatives with respect to $\delta$, we get for $1 \leq l^{\prime} \leq L$ and $1 \leq t^{\prime} \leq T$ :

$$
\sum_{j=1}^{J_{t^{\prime}}} \int\left\{Y_{t^{\prime}, j}-\sum_{l=0}^{L} \widehat{Z}_{t^{\prime}, l} \widehat{m}_{l}(u)\right\} \widehat{m}_{l^{\prime}}(u) K_{h}\left(u-X_{t^{\prime}, j}\right) d u=0 .
$$

Using the following notation, for $1 \leq t \leq T$

$$
\begin{gathered}
\widehat{p}_{t}(u)=\frac{1}{J_{t}} \sum_{j=1}^{J_{t}} K_{h}\left(u-X_{t, j}\right), \\
\widehat{q}_{t}(u)=\frac{1}{J_{t}} \sum_{j=1}^{J_{t}} K_{h}\left(u-X_{t, j}\right) Y_{t, j},
\end{gathered}
$$

from (12) - (13) we get, for $1 \leq l^{\prime} \leq L$ and $1 \leq t \leq T$ :

$$
\begin{gathered}
\sum_{t=1}^{T} J_{t} \widehat{Z}_{t, l^{\prime}} \widehat{q}_{t}(u)=\sum_{t=1}^{T} J_{t} \sum_{l=0}^{L} \widehat{Z}_{t, l^{\prime}} \widehat{Z}_{t, l} \widehat{p}_{t}(u) \widehat{m}_{l}(u) \\
\int \widehat{q}_{t}(u) \widehat{m}_{l^{\prime}}(u) d u=\sum_{l=0}^{L} \widehat{Z}_{t, l} \int \widehat{p}_{t}(u) \widehat{m}_{l^{\prime}}(u) \widehat{m}_{l}(u) d u .
\end{gathered}
$$

The algorithm exploits equations (16) and (17) iteratively:

1. For an appropriate initialisation of $\widehat{Z}_{t, l}^{(0)}, t=1, \ldots, T, l=1, \ldots, L$ get an initial estimate of $\widehat{m}^{(0)}=\left(\widehat{m}_{0}, \ldots, \widehat{m}_{L}\right)^{\top}$

2. Update $\widehat{Z}_{t, l}^{(1)}, t=1, \ldots, T$

3. Estimate $\widehat{m}^{(1)}$ 


\section{Go to step 2,}

until minor changes occur during the cycle.

The choice of the model size $L$ is based on computing the residual sum of squares:

$$
R V(L)=\frac{\sum_{t} \sum_{j}\left\{Y_{t, j}-\sum_{l=0}^{L} \widehat{Z}_{t, l} \widehat{m}_{l}\left(X_{t, j}\right)\right\}^{2}}{\sum_{t} \sum_{j}\left\{Y_{t, j}-\bar{Y}\right\}^{2}},
$$

where $\bar{Y}$ is the overall mean of the observation. We choose $L$ such that the proportion of variation explained by the model among the total variation $1-R V(L)$ is sufficiently high.

For the data-driven choice of bandwidths we take weighted AIC, as Fengler et al. (2007) did. The two criteria are:

$\Xi_{A I C_{1}}=\frac{1}{N} \sum_{t, j}\left\{Y_{t, j}-\sum_{l=0}^{L} \widehat{Z}_{t, l} \widehat{m}_{l}\left(X_{t, j}\right)\right\}^{2} w\left(X_{t, j}\right) \exp \left\{\frac{2 L}{N} K_{h}(0) \int w(u) d u\right\}$

and

$$
\Xi_{A I C_{2}}=\frac{1}{N} \sum_{t, j}\left\{Y_{t, j}-\sum_{l=0}^{L} \widehat{Z}_{t, l} \widehat{m}_{l}\left(X_{t, j}\right)\right\}^{2} \exp \left\{\frac{2 L}{N} K_{h}(0) \frac{\int w(u) d u}{\int w(u) p(u) d u}\right\} .
$$

For our implementation, we consider as outliers contracts with maturity less than 10 days, since their behaviour is irregular due to the expiry effect. We also remove observations with implied volatility smaller than 0.04 or lager than 0.8. The data set contains 829299 observations in the KOSPI 200 option data (about 3371 per day) and 1113845 observations in the ODAX data (about 4546 per day). Log-implied volatilities are modeled on moneyness and time to maturity $\left(\kappa_{t, j}, \tau_{t, j}\right)$. The grid covers moneyness $\kappa \in[0.8,1.2]$ and time to maturity $\tau \in[0.02,0.5]$ measured in years. $L=3$ factor functions are employed for both markets and bandwidths $(0.02,0.04)$ for the KOSPI 200 option and $(0.02,0.05)$ for the ODAX have been chosen, according to variance explained, $\Xi_{A I C_{1}}$ and $\Xi_{A I C_{2}}$ criterions, see Table 1 . We have recalculated the model with the same bandwidths with $2,3,4$ and 5 dynamic factor functions, see Table 2 . A choice of 3 factor functions is based on a balance between model economy and efficiency - capturing as many explained variances as possible while keeping a small number of factors.

Figure 3 displays Model fits on 17 September, 2003 for both markets. The IVS of the KOSPI 200 option is flatter than the ODAX on this day. Figure 4 shows factor functions $\widehat{m}_{i}, i=1,2,3 . \widehat{m}_{1}$ is positive in both markets. They 


\begin{tabular}{c|cccc}
\hline \hline & Bandwidths & $1-R V(3)$ & $\Xi_{A I C_{1}}$ & $\Xi_{A I C_{2}}$ \\
\hline KOSPI 200 option & $(0.02,0.04)$ & 0.881 & 0.00106 & 0.00570 \\
\hline ODAX & $(0.02,0.05)$ & 0.921 & 0.00172 & 0.00612 \\
\hline \hline
\end{tabular}

Table 1: DSFM modelling result.

\begin{tabular}{cc|cc}
\hline \hline$L$ & & $1-R V(L)$ & $\Delta R V$ \\
\hline 2 & KOSPI 200 Option & 0.833 & \\
& ODAX & 0.886 & \\
\hline 3 & KOSPI 200 Option & 0.881 & 0.048 \\
& ODAX & 0.921 & 0.035 \\
\hline 4 & KOSPI 200 Option* & 0.900 & 0.019 \\
& ODAX & 0.942 & 0.021 \\
\hline 5 & KOSPI 200 Option** & 0.880 & -0.020 \\
& ODAX & 0.955 & 0.013 \\
\hline \hline
\end{tabular}

Table 2: Explained variance for the model size. *Bandwidth (0.02, 0.06). **Bandwidth (0.02, 0.14).

are both relatively flat. Factor $\widehat{m}_{1}$ can be interpreted as the time dependent mean of the (log-)IVS, a shift effect. $\widehat{m}_{2}$ for the ODAX tends to trend upward in the moneyness axis, from negative to positive. The surface is close to 0 when moneyness is near 1 . This trend is strong when time to maturity is small while it becomes weak when time to maturity increases. For $\widehat{m}_{3}$ of the KOSPI 200 option, a downward trend exists in maturity direction when moneyness is in the region of 0.8 . The surface slopes down when time to maturity increases. This trend becomes weak when moneyness increases. $\widehat{m}_{3}$ of the ODAX appears to smile in the maturity direction.

We interpret the effect of the first factor loadings such that $\widehat{Z}_{1}$ represents the overall level shift of the IVS. We observe in Figure 5 that $\widehat{Z}_{1}$ of both markets seem to have a common trend. $\widehat{Z}_{2}$ of the KOSPI 200 option has stronger fluctuations than that of the ODAX. 

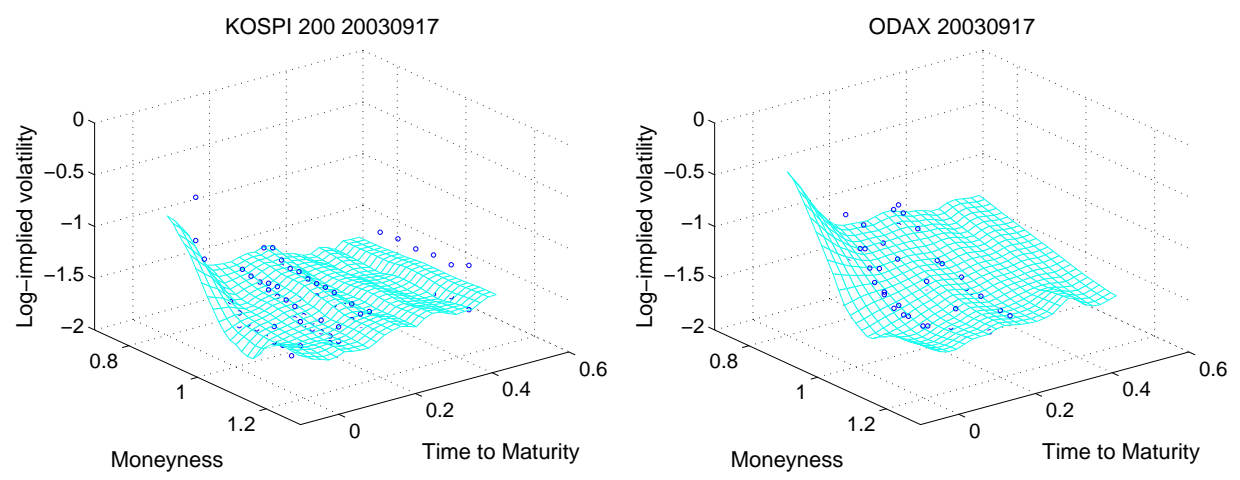

Figure 3: DSFM fit for KOSPI 200 option (left) with bandwidths (0.02, 0.04) and the ODAX (right) with bandwidths (0.02, 0.05) on 17 September, 2003.

\section{Joint dynamics of KOSPI 200 option and ODAX}

Let the $k$ dimensional vector series $\widehat{Z}_{t}=\left(\widehat{Z}_{1 t}, \ldots, \widehat{Z}_{k t}\right)^{\top}, t=0, \ldots, T$ follow a $\operatorname{VAR}(p)$ model:

$$
\widehat{Z}_{t}=v+\sum_{j=1}^{p} \Phi_{j} \widehat{Z}_{t-j}+\varepsilon_{t}
$$

where $v=\left(v_{1}, \ldots, v_{k}\right)^{\top}$ is a $k \times 1$ vector of intercepts, $\Phi_{j}$ are $k \times k$ parameter matrices and the error process $\varepsilon_{t}=\left(\varepsilon_{1 t}, \ldots, \varepsilon_{k t}\right)^{\top}$ is such that

$$
E\left[\varepsilon_{t}\right]=0 \text { and } E\left(\varepsilon_{t} \varepsilon_{t-k}^{\top}\right)= \begin{cases}0 & \text { for } k \neq 0 \\ \Sigma & \text { for } k=0\end{cases}
$$

$\Sigma$ is a time invariant positive definite variance-covariance matrix of the innovation series. In this setting we acknowledge cross dependencies between the $\widehat{Z}_{t}$ series, i.e., each $\widehat{Z}_{t}$ depends on its "history" and the "history" of the other series in the system. Therefore the VAR model posits a set of relationships between past lagged values of all variables in the model and the current value of each variable in the model. Descriptive statistics for the factor loadings of our model are presented in Table 3. We denote the dynamic system of factor loadings by

$$
\widehat{Z}_{t}=\left(\widehat{Z}_{t, 1}^{k}, \widehat{Z}_{t, 2}^{k}, \widehat{Z}_{t, 3}^{k}, \widehat{Z}_{t, 1}^{d}, \widehat{Z}_{t, 2}^{d}, \widehat{Z}_{t, 3}^{d}\right)^{\top},
$$

where the superscripts $k$ and $d$ denote loadings of KOSPI and ODAX, respectively. Unit root tests using the augmented Dickey-Fuller (ADF) and the Elliott et al. (ERS) testing strategies are implemented to determine 

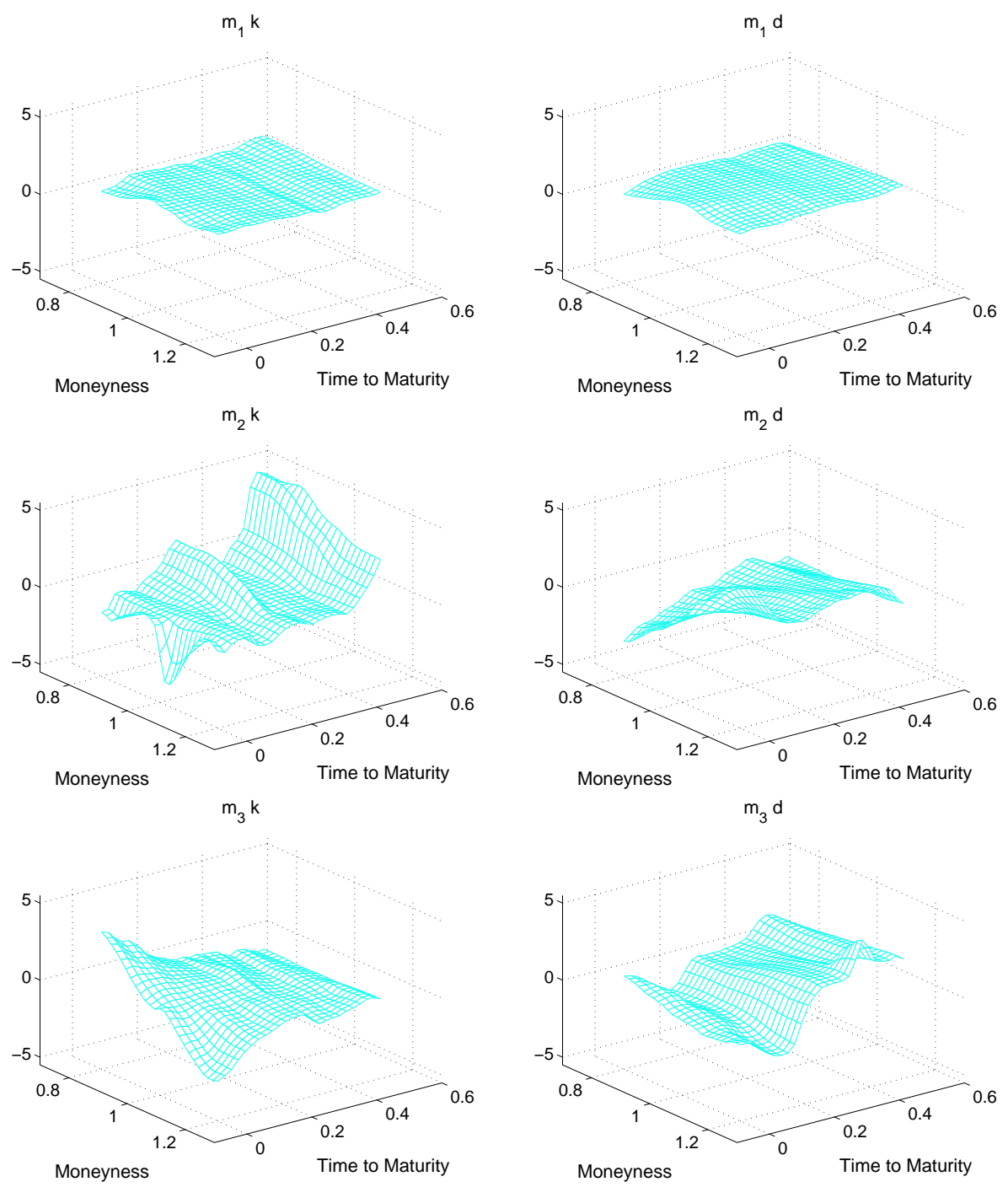

Figure 4: Estimated factor function $\widehat{m}_{l}$ for KOSPI 200 option (left) and for ODAX (right) from 2 January, 2003 to 30 December, 2003.

\begin{tabular}{cc|ccccccc}
\hline \hline & & Mean & Median & Min. & Max. & Std. & Kurt. & Skew. \\
\hline KOSPI 200 option & $\widehat{Z}_{1}$ & -1.22 & -1.28 & -1.57 & -0.88 & 0.18 & 1.59 & 0.06 \\
& $\widehat{Z}_{2}$ & 0.00 & 0.00 & -0.5 & 0.62 & 0.09 & 19.16 & 1.29 \\
& $\widehat{Z}_{3}$ & 0.00 & 0.00 & -0.19 & 0.21 & 0.08 & 2.45 & -0.19 \\
\hline \multirow{2}{*}{ ODAX } & $\widehat{Z}_{1}$ & -0.93 & -0.98 & -1.23 & -0.56 & 0.18 & 1.94 & 0.49 \\
& $\widehat{Z}_{2}$ & 0.00 & 0.00 & -0.12 & 0.17 & 0.06 & 2.66 & 0.18 \\
& $\widehat{Z}_{3}$ & 0.00 & -0.01 & -0.18 & 0.22 & 0.05 & 4.52 & 0.48 \\
\hline \hline
\end{tabular}

Table 3: Descriptive statistics for factor loadings $\widehat{Z}_{1}, \widehat{Z}_{2}$ and $\widehat{Z}_{3}$. 
$\mathrm{Z1}$

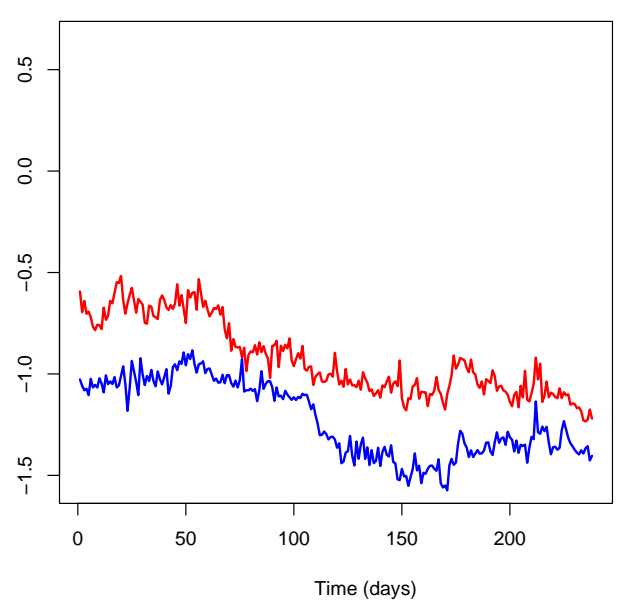

$\mathrm{Z2}$

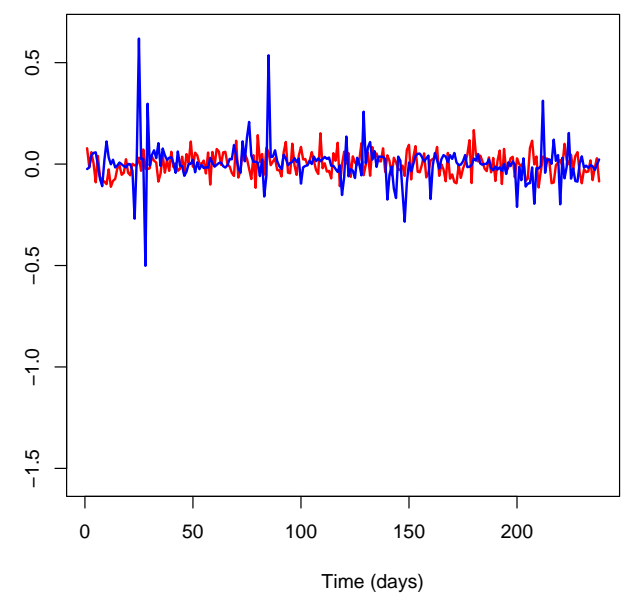

Z3

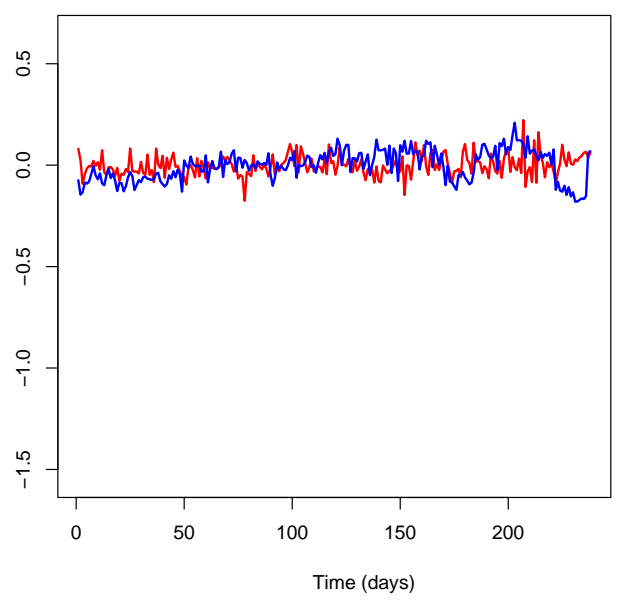

Figure 5: Time series of factor loadings $\widehat{Z}_{1}, \widehat{Z}_{2}$ and $\widehat{Z}_{3}$ for KOSPI 200 option (blue) and ODAX (red) from 2 January, 2003 to 30 December, 2003. 


\begin{tabular}{c|ccc|ccc}
\hline \hline & \multicolumn{3}{|c|}{ KOSPI 200 option } & \multicolumn{3}{c}{ ODAX } \\
\hline & $\widehat{Z}_{1}^{k}$ & $\widehat{Z}_{2}^{k}$ & $\widehat{Z}_{3}^{k}$ & $\widehat{Z}_{1}^{d}$ & $\widehat{Z}_{2}^{d}$ & $\widehat{Z}_{3}^{d}$ \\
\hline ADF-AIC & -1.074 & $-15.129^{* *}$ & $-3.409^{*}$ & -0.880 & $-16.605^{* *}$ & $-6.686^{* *}$ \\
$\widehat{b}$ & 5 & 0 & 2 & 4 & 0 & 2 \\
\hline ADF-HQ & -1.039 & $-15.129^{* *}$ & $-3.409^{*}$ & -0.880 & $-16.605^{* *}$ & $-8.656^{* *}$ \\
$\widehat{b}$ & 3 & 0 & 2 & 4 & -0 & 1 \\
\hline ERS-AIC & 23.914 & $0.225^{* *}$ & $2.947^{*}$ & 55.112 & $0.740^{* *}$ & $1.313^{* *}$ \\
$\widehat{b}$ & 5 & 0 & 2 & 4 & 0 & 2 \\
\hline ERS-HQ & 24.783 & $0.225^{* *}$ & $2.947^{*}$ & 55.112 & $0.740^{* *}$ & $0.952^{* *}$ \\
$\widehat{b}$ & 3 & 0 & 2 & 4 & 0 & 1 \\
\hline \hline
\end{tabular}

Table 4: Unit root tests for factor loadings $\widehat{Z}_{1}, \widehat{Z}_{2}$ and $\widehat{Z}_{3}$. Tests include constant. ADF-AIC and ERS-HQ refer to the ADF tests using AIC criteria and ERS tests using $H Q$ criteria to estimate the lag length $b$ chosen for the estimation regression of the autoregressive spectral density estimator. Critical values for ADF test are-3.458(1\%), -2.874(5\%) and-2.573(10\%). Critical values for ERS test are 1.925(1\%), 3.187(5\%) and 4.359(10\%). ** and ${ }^{*}$ denote significance at the $1 \%$ and $5 \%$ level, respectively.

whether the individual series are trend stationary or difference stationary, see Table 4. We apply standard information criteria as AIC, SC and HQ to select the lag order $p$ for our VAR. These criteria balance the trade-off between model fit and the number of parameters to be estimated and allow a fairly parsimonious model specification. We use $p=2$ lags as suggested by the AIC, since we want to model the dynamic relations between the factor loading series by impulse response functions in a more flexible way.

Using the lag selection criteria and the parsimony principle, a $\operatorname{VAR}(2)$ model $\widehat{Z}_{t}=\left(\Delta \widehat{Z}_{t, 1}^{k}, \widehat{Z}_{t, 2}^{k}, \widehat{Z}_{t, 3}^{k}, \Delta \widehat{Z}_{t, 1}^{d}, \widehat{Z}_{t, 2}^{d}, \widehat{Z}_{t, 3}^{d}\right)^{\top}$, where $\widehat{Z}_{t, 1}^{k}$ and $\widehat{Z}_{t, 1}^{d}$ enter as first order differences, is chosen to analyse the system. The residual correlation matrix $\widehat{\Sigma}$, given in (22), gives us some useful information about the contemporaneous correlation structure, i.e. if there is overlapping information between variables. This information has implications for interpretation of 


\begin{tabular}{lccccc}
\hline \hline Model & $p$ & $\mathrm{Q}(24)$ & LMF(3) & LBJ & ARCH(1) \\
\hline VAR & 2 & 0.74 & 0.25 & 0.00 & 0.00 \\
\hline \hline
\end{tabular}

Table 5: p-values for VAR(2) diagnostic tests. Q(24) denotes an adjusted portmanteau test involving 24 autocorrelation matrices, LMF(3) is LM test for autocorrelation of order 3. LBJ represents the multivariate LomnickiJaque-Bera test for nonnormality and $A R C H(1)$ is the multivariate firstorder ARCH test.

the results of the impulse responses.

$$
\widehat{\Sigma}=\left(\begin{array}{cccccc}
\Delta \widehat{Z}_{1}^{k} & \widehat{Z}_{2}^{k} & \widehat{Z}_{3}^{k} & \Delta \widehat{Z}_{1}^{d} & \widehat{Z}_{2}^{d} & \widehat{Z}_{3}^{d} \\
& -0.11 & 0.17 & 0.13 & 0.02 & -0.12 \\
& 1 & 0.008 & -0.04 & 0.005 & 0.005 \\
& & 1 & 0.06 & 0.07 & -0.04 \\
& & & 1 & 0.04 & 0.01 \\
& & & & 1 & 0.09 \\
& & & & & 1
\end{array}\right)
$$

We apply a number of standard diagnostic tests to check the adequacy of the model, see Table 5 . While the estimated residuals are free from autocorrelation, normality and conditional homoscedasticity are rejected. Considering that the VAR is a rough approximation of the dynamics of the system, a more detailed analysis to accommodate the $\mathrm{ARCH}$ effects is beyond the scope of this paper.

The dynamic relations between the factors are captured by the impulse response functions. Figures 6 and 7 give the generalised impulse responses to a positive shock in the loadings series of both markets. The results indicate that there is a positive but small response between the overall changes in the risk levels of the German and Korean markets. $\Delta \widehat{Z}_{1}^{k}$ responds only slightly positive for about 1-2 days after a shock in $\Delta \widehat{Z}_{1}^{d}$. On the other hand the response of $\Delta \widehat{Z}_{1}^{d}$ to a shock in $\Delta \widehat{Z}_{1}^{k}$ is barely positive. We take caution in interpreting the results since $\widehat{Z}_{1}^{k}$ and $\widehat{Z}_{1}^{d}$ enter the VAR model in first differences. We therefore interpret that an increase in the DAX volatility translates into a small amount of uncertainty in the Korean market. On the other hand effects of shock from the KOSPI do not translate into effective uncertainty in the DAX. Our results suggest that a shock from one market is likely to work through the other market's system and may not induce greater impulse responses either way. This may also reflect the view of noarbitrage relation between the two markets. We have conducted Granger 


\begin{tabular}{c|c}
\hline \hline$H_{0}$ & Test result \\
\hline$\Delta \widehat{Z}_{1}^{d} \nrightarrow \Delta \widehat{Z}_{1}^{k}, \widehat{Z}_{2}^{k}, \widehat{Z}_{3}^{k}$ & $F(6,900)=1.70[0.12]$ \\
$\Delta \widehat{Z}_{1}^{k} \nrightarrow \Delta \widehat{Z}_{1}^{d}, \widehat{Z}_{2}^{k}, \widehat{Z}_{3}^{k}$ & $F(6,900)=0.93[0.46]$ \\
$\Delta \widehat{Z}_{1}^{k} \nrightarrow \Delta \widehat{Z}_{1}^{d}, \widehat{Z}_{2}^{d}, \widehat{Z}_{3}^{d}$ & $F(6,900)=1.15[0.32]$ \\
$\Delta \widehat{Z}_{1}^{d} \nrightarrow \Delta \widehat{Z}_{1}^{k}, \widehat{Z}_{2}^{d}, \widehat{Z}_{3}^{d}$ & $F(6,900)=2.62[0.02]$ \\
$\Delta \widehat{Z}_{1}^{d} \nrightarrow \Delta \widehat{Z}_{1}^{k}$ & $F(4,446)=4.74[0.02]$ \\
$\Delta \widehat{Z}_{1}^{k} \nrightarrow \Delta \widehat{Z}_{1}^{d}$ & $F(4,446)=0.68[0.60]$ \\
\hline \hline
\end{tabular}

Table 6: Granger causality tests for VAR(2). $\nrightarrow$ denotes "does not Granger cause". p-values are given in brackets. Critical values are 3.32(1\%), $2.37(5 \%)$ 1.94(10\%) for $F(4,446)$ and $2.80(1 \%)$, 2.10(5\%), $1.77(10 \%)$ for $F(6,900)$.

causality tests to further investigate the relationship between these variables, see Table 6. Granger non-causality of $\Delta \widehat{Z}_{1}^{d}$ for $\Delta \widehat{Z}_{1}^{k}, \widehat{Z}_{2}^{d}, \widehat{Z}_{3}^{d}$ and of $\Delta \widehat{Z}_{1}^{d}$ for $\Delta \widehat{Z}_{1}^{k}$ is rejected at $5 \%$ significance level. We observe that Granger noncausality from $\Delta \widehat{Z}_{1}^{k}$ to $\Delta \widehat{Z}_{1}^{d}$ is not rejected. This indicates a unidirectional effect from the ODAX to the KOSPI 200 option market.

Due to the appearance of a common trend, we next investigated the cointegration relationship between $\widehat{Z}_{1}^{k}$ and $\widehat{Z}_{1}^{d}$. The trace and maximum eigenvalue tests of cointegration are applied to determine whether or not the KOSPI 200 option and the ODAX first loading series share a common stochastic trend. The test results in Table 7 show that both tests fail to reject cointegration between the factor loading series $\widehat{Z}_{1}^{d}$ and $\widehat{Z}_{1}^{k}$.

The existence of cointegration means that there is a long-term relationship between two variables and that they are influenced by the same stochastic trend. Since $\widehat{Z}_{1}$ is the loading for $\widehat{m}_{1}$, which is thought to be the time dependent mean of the (log-)IVS, a long-term relationship between two markets' first loadings could probably indicate a long-term relationship between two markets' implied volatility surfaces. From a theoretical point of view, this result is not surprising. According to the no-arbitrage relation, for global exchanges like KRX and Deutsche Börse/Eurex, there should be no arbitrage opportunity between them. Implied volatility as a state indicator for a market situation, should also reflect this no-arbitrage relation in the long term. Consequently, when global markets have large fluctuations, so do KRX and Deutsche Börse/Eurex; implied volatilities of these two markets will become large and vice versa. 

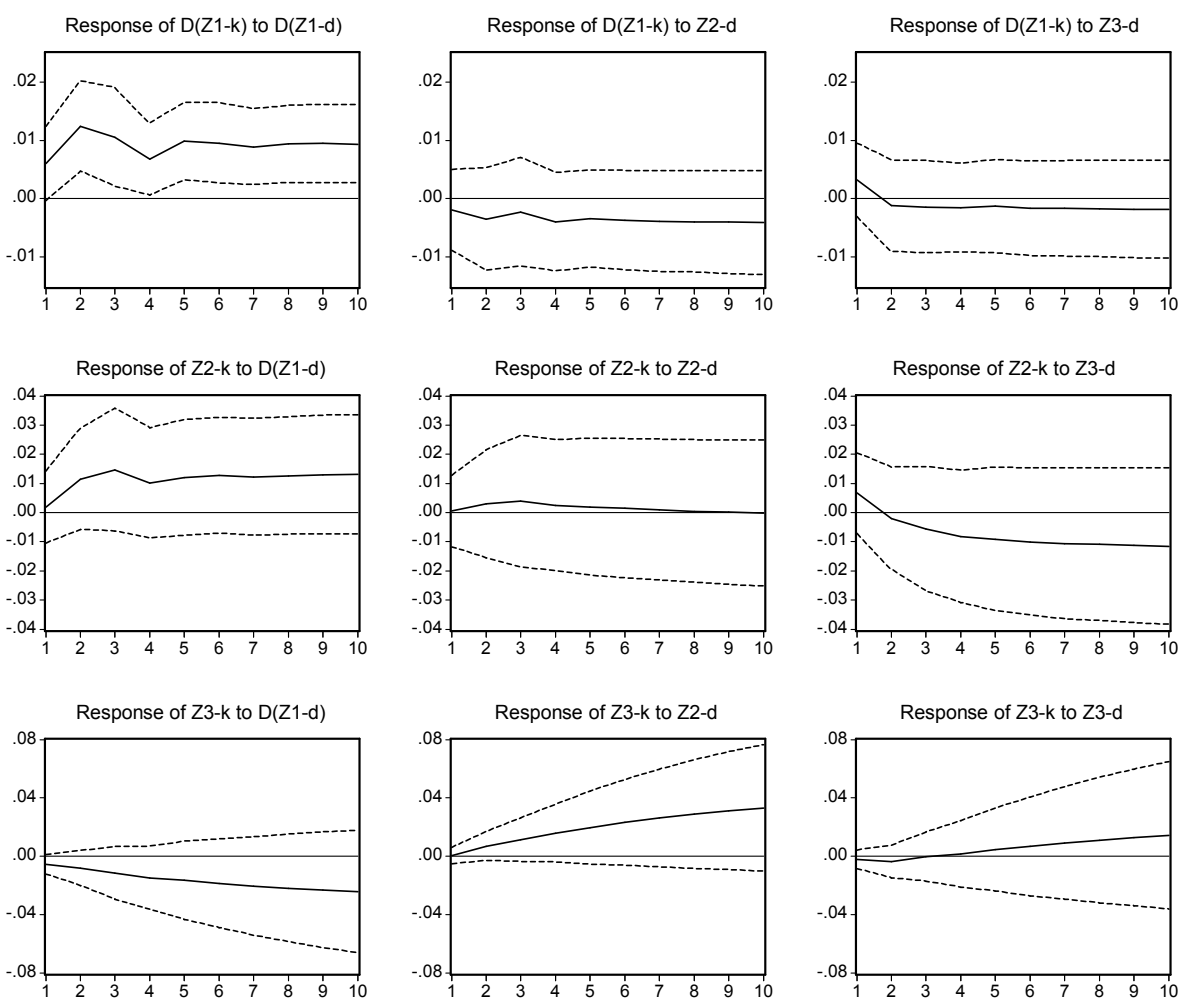

Figure 6: Generalized impulse responses of the KOSPI 200 option factor loadings to the ODAX factor loadings obtained from a VAR(2). Dashed lines indicate approximate $95 \%$ confidence intervals. $D(Z 1-k)$ and $D(Z 1-d)$ denote the first differences of the first loadings, $\Delta \widehat{Z}_{1}^{k}$ and $\Delta \widehat{Z}_{1}^{d}$ respectively.

\begin{tabular}{c|c|c|c||c|c|c|c}
\hline \hline \multicolumn{4}{c||}{ Trace test } & \multicolumn{4}{c}{ Maximum eigenvalue test } \\
\hline Rank & Test statistic & $5 \%$ critical value & p-value & Rank & Test statistic & $5 \%$ critical value & p-value \\
\hline 1 & 2.60 & 9.16 & 0.66 & 1 & 2.60 & 9.16 & 0.66 \\
0 & 21.48 & 20.26 & 0.03 & 0 & 18.88 & 15.89 & 0.02 \\
\hline \hline
\end{tabular}

Table 7: Two cointegration tests for the KOSPI 200 option $\widehat{Z}_{1}^{k}$ and the ODAX $\widehat{Z}_{1}^{d}$, constant included. Lag order is 1 in the VECM. 

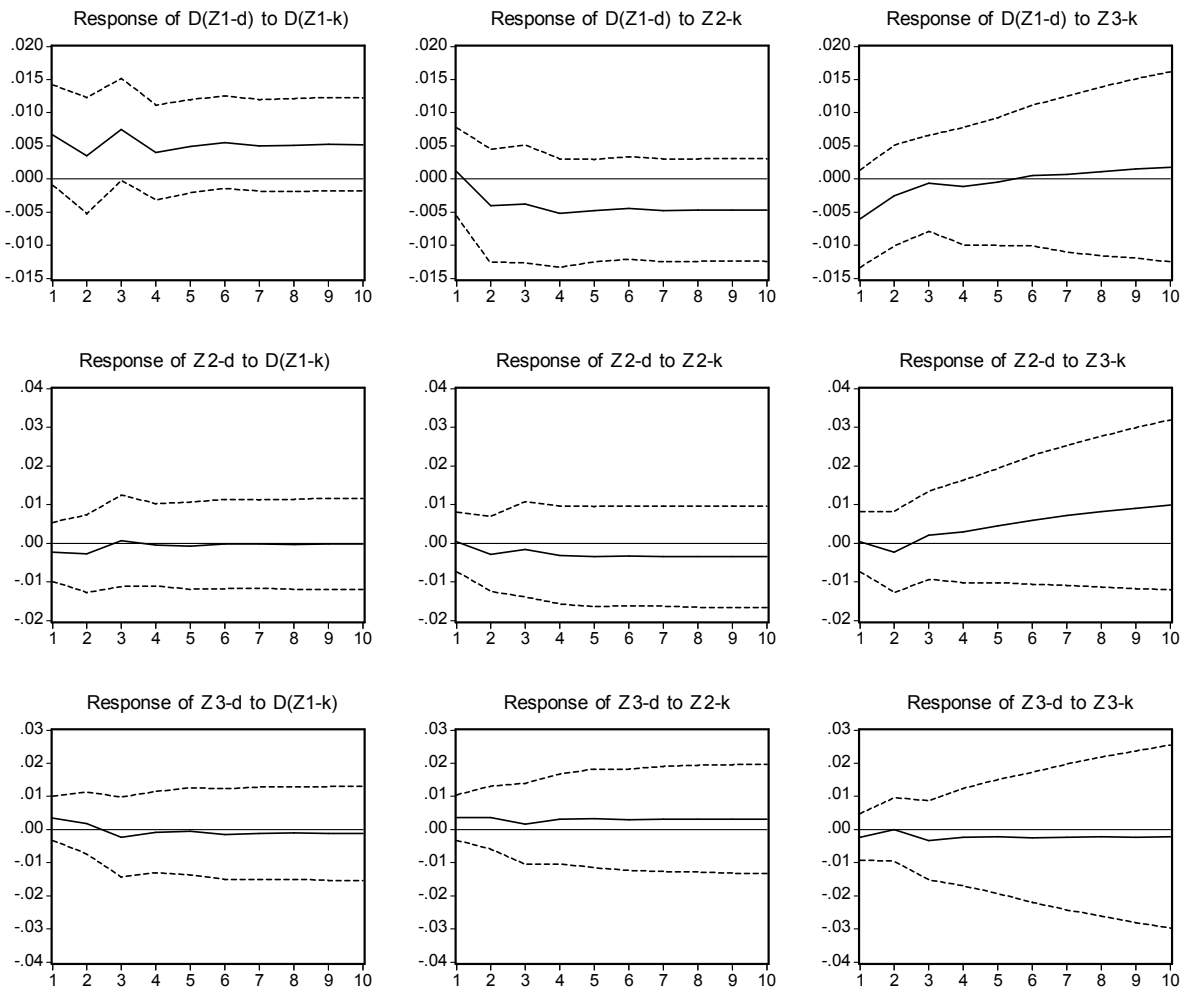

Figure 7: Generalized impulse responses of the ODAX factor loadings to the KOSPI 200 option factor loadings obtained from a VAR(2). Dashed lines indicate approximate $95 \%$ confidence intervals. $D(Z 1-k)$ and $D(Z 1-d)$ denote the first differences of the first loadings, $\Delta \widehat{Z}_{1}^{k}$ and $\Delta \widehat{Z}_{1}^{d}$ respectively. 


\section{Conclusion}

In this paper we analyse implied volatility as a function of the strike and time to maturity using recent developments in dimension reduction techniques, Dynamic Semiparametric Factor Models (DSFM), which allow for the estimation of the implied volatility surface in a dynamic context. As proxy for volatility, factor loading series derived from the DSFM application on option prices from the German and Korean markets are jointly analysed. We examine volatility transmission between the markets within the framework of a vector autoregression (VAR) approach.

An important choice for analysis in the VAR framework relates to the form (level or first difference) of the variables involved. Unit root tests are applied to check whether the VAR model is estimated in levels or in first differences. The first loading series of the two markets contain unit root and follow a common long term stochastic trend, i.e. are cointegrated. Estimation of an vector error correction model (VECM) is theoretically beneficial (Engle and Granger (1987)), but their performance is contested at short horizon, Naka and Tufte (1997). We therefore estimated the VAR model using stationary transformation (first difference) of the loading series that exhibit unit roots.

Interpretations of our results are based on the impulse response and Granger causality analysis. Our results show that a shock in the volatility of one market may not translate directly into greater uncertainty in another market. Furthermore, as a result of cointegration, it is unlikely that portfolio investors may benefit from diversification among these markets.

\section{References}

Ahoniemi, K. and Lanne, M. (2007). Joint Modeling of Call and Put Implied Volatility. Discussion Paper 198, Helsinki Center of Economic Research, University of Helsinki, Finland.

Black, F. and Scholes, M. (1973). The pricing of options and corporate liabilities. Journal of Political Economy, 81:637-654.

Borak, S., Härdle, W., and Fengler, M. R. (2005). DSFM fitting of Implied Volatility Surfaces. Proceedings 5th International Conference on Intelligent, System Design and Applications, IEEE Computer Society Number P2286, Library of Congress Number 2005930524.

Brüggemann, R., Härdle, W. K., Mungo, J., and Trenkler, C. (2008). VAR 
Modeling for Dynamic Loadings Driving Volatility Strings. Journal of Financial Econometrics, 6,3:361-368.

Engle, R. F. and Granger, C. W. J. (1987). Co-integration and error correction: Representation, estimation, and testing. Econometrica, 55:251-276.

Fengler, M. R., Härdle, W. K., and Mammen, E. (2007). A Dynamic Semiparametric Factor Model for Implied Volatility String Dynamics. Journal of Financial Econometrics, 5,2:189-218.

Franke, J., Härdle, W., and Hafner, C. (2008). Statistics of Financial Markets: An introduction. Springer, Heidelberg, 2nd edition.

Härdle, W., Klinke, S., and Müller, M. (2000). XploRe - Learning Guide. Springer, Heidelberg.

Härdle, W., Müller, M., Sperlich, S., and Werwatz, A. (2003). Non- and Semiparametric Models. Springer, Heidelberg.

Härdle, W. and Simar, L. (2007). Applied Multivariate Statistical Analysis. Springer, Heidelberg, 2nd edition.

Hull, J. C. (2000). Options, Futures and other Derivatives. Prentice Hall.

Lütkepohl, H. (2005). New Introduction to Multiple Time Series Analysis. Springer, Berlin.

Naka, A. and Tufte, D. (1997). Examining impulse response functions in cointegrated systems. Applied Economics, 29:1593-1603.

Park, B., Mammen, E., Härdle, W., and Borak, S. (2009). Dynamic semiparametric factor models. Journal of the American Statistical Association, forthcoming.

Tsay, R. S. (2002). Analysis of Financial Time Series. Wiley. 


\section{SFB 649 Discussion Paper Series 2009}

For a complete list of Discussion Papers published by the SFB 649, please visit http://sfb649. wiwi. hu-berlin.de.

001 "Implied Market Price of Weather Risk" by Wolfgang Härdle and Brenda López Cabrera, J anuary 2009.

002 "On the Systemic Nature of Weather Risk" by Guenther Filler, Martin Odening, Ostap Okhrin and Wei Xu, January 2009.

003 "Localized Realized Volatility Modelling" by Ying Chen, Wolfgang Karl Härdle and Uta Pigorsch, January 2009.

004 "New recipes for estimating default intensities" by Alexander Baranovski, Carsten von Lieres and André Wilch, January 2009.

005 "Panel Cointegration Testing in the Presence of a Time Trend" by Bernd Droge and Deniz Dilan Karaman Örsal, January 2009.

006 "Regulatory Risk under Optimal Incentive Regulation" by Roland Strausz, January 2009.

007 "Combination of multivariate volatility forecasts" by Alessandra Amendola and Giuseppe Storti, January 2009.

008 "Mortality modeling: Lee-Carter and the macroeconomy" by Katja Hanewald, January 2009.

009 "Stochastic Population Forecast for Germany and its Consequence for the German Pension System" by Wolfgang Härdle and Alena Mysickova, February 2009.

010 "A Microeconomic Explanation of the EPK Paradox" by Wolfgang Härdle, Volker Krätschmer and Rouslan Moro, February 2009.

011 "Defending Against Speculative Attacks" by Tijmen Daniëls, Henk Jager and Franc Klaassen, February 2009.

012 "On the Existence of the Moments of the Asymptotic Trace Statistic" by Deniz Dilan Karaman Örsal and Bernd Droge, February 2009.

013 "CDO Pricing with Copulae" by Barbara Choros, Wolfgang Härdle and Ostap Okhrin, March 2009.

014 "Properties of Hierarchical Archimedean Copulas" by Ostap Okhrin, Yarema Okhrin and Wolfgang Schmid, March 2009.

015 "Stochastic Mortality, Macroeconomic Risks, and Life Insurer Solvency" by Katja Hanewald, Thomas Post and Helmut Gründl, March 2009.

016 "Men, Women, and the Ballot Woman Suffrage in the United States" by Sebastian Braun and Michael Kvasnicka, March 2009.

017 "The Importance of Two-Sided Heterogeneity for the Cyclicality of Labour Market Dynamics" by Ronald Bachmann and Peggy David, March 2009.

018 "Transparency through Financial Claims with Fingerprints - A Free Market Mechanism for Preventing Mortgage Securitization Induced Financial Crises" by Helmut Gründl and Thomas Post, March 2009.

019 "A Joint Analysis of the KOSPI 200 Option and ODAX Option Markets Dynamics" by Ji Cao, Wolfgang Härdle and J ulius Mungo, March 2009.

\section{SFB 649, Spandauer Straße 1, D-10178 Berlin} http:/ / sfb649.wiwi.hu-berlin.de 